\title{
Introduction to the Symposium
}

\author{
Brian A. Burt, BDS, MPH, PhD
}

\begin{abstract}
January 25,1995 , is the 50th anniversary of the first controlled addition of fluoride to a public water supply. Those 50 years have seen extraordinary advances in oral health and consequent quality of life, for which fluoride use is generally considered the primary reason. More extensive exposure to fluoride in the modern era, however, has led to both a continuing decline in caries experience and an increased prevalence of dental fluorosis in children. At the other end of life, fluoride's role in bone strength among older people is not well defined. This symposium examines several aspects of fluoride use in the United States today, and has the purpose of helping to define the balance between maximizing the benefits of fluoride while minimizing its undesirable side effects. (J Public Health Dent 1995;55(1):37-8]
\end{abstract}

Key Words: symposium, fluoride, fluorosis, bone, caries.

On January 25, 1995, we recognize the 50th anniversary of the first controlled addition of fluoride to a public water supply in Grand Rapids, Michigan. Any 50 th anniversary is a time for both celebration and reflection, so while we take justifiable pride in the achievements of those 50 years, it is also an appropriate time to review the status of fluoride in public health. This symposium is conducted in the knowledge that fluoride has produced extraordinary benefits in oral health and the quality of life for many millions of people. At the same time, it is the ongoing responsibility of the dental profession, and especially of its dental public health community, to constantly reassess our use of fluoride so that we are always making the best possible use of current research knowledge. The title of this symposium has been paraphrased from a 1991 report by Dr. James Mason (1), the assistant secretary for health at the time, which raised the question of how much fluoride is needed to maintain its oral health benefits while minimizing any less desirable side effects. This symposium is intended to stimulate our thinking about achieving that balance.

Water fluoridation began with justifiable excitement and enthusiasm after years of painstaking research; but even the most avid supporter of the measure in 1945 could not have anticipated the extraordinary improvements in oral health that have been realized since then. Caries prevalence and severity in our children have reached the lowest levels yet recorded, and still with little indication that we have reached rock bottom (at least in the permanent dentition). While we are aware that the reductions in caries experience have not been uniform across all segments of society, we are still in a position to speculate happily on what the lowest levels of community caries experience might be. At the other end of the age spectrum, fewer older adults than ever are becoming edentulous, and dentate people are retaining more of their teeth than ever before.

Perhaps the greatest gains in oral health have been in areas not measured by our usual indexes: whole generations of young people have never suffered toothache, and broad toothy smiles enhance self-esteem and self-confidence. One of the happiest challenges in teaching is trying to get across to new generations of students just what caries looked like in the old days. I think back on the trauma of my own early dental treatment experi- ences while my own children, now in their mid-20s, ask me what "a shot in the mouth" feels like!

Although a number of social and technological changes over the last 50 years have contributed toward this massive improvement in oral health, most consider the widespread use of fluoride to be the primary cause. Our current view of how fluoride works to prevent the development of caries is that the maintenance of elevated fluoride levels in the oral environment, especially in dental plaque, is a major factor in the prevention of clinically evident lesions. This model has retained some aspects of the original assumptions on the preeruptive value of fluoride, but now ascribes more weight to the inhibition of demineralization and promotion of remineralization. The more that new knowledge on fluoride becomes available, the more fluoride's continuing key role in caries prevention is underscored. The generally low levels of caries we see today is no reason to turn away from fluoride programs, though program selection today requires more careful thought than it ever did.

Exposure to fluoride in the modern world has expanded far beyond the degree envisaged by the fluoride pioneers of 50 or more years ago. They dealt almost exclusively with fluoride in drinking water, and their careful research on the appropriate concentrations was conducted from that viewpoint. Controlled water fluoridation was followed within a few years by the introduction of fluoride dietary supplements; professionally applied topical solutions and gels soon followed. Fluoride toothpastes first appeared in the late 1950 s and within a generation had come to dominate the toothpaste market. Fluoride mouthrinses and school water fluoridation emerged as public health programs in the 1970s, high-fluoride gels became available

This paper and those following were presented at the annual meeting of the AAPHD in New Orleans, LA, October 1994. Dr. Burt is director of the Program in Dental Public Health, School of Public Health, University of Michigan, Ann Arbor, MI 48109-2029. Internet: bburt@umich.edu. Reprints will not be available. 
for home use by prescription, and many of the cosmetic mouthwashes sold over the counter had fluoride added to them. Perhaps the most significant new exposure came with the processing of foods and beverages with fluoridated water. Because processed foods and drinks rather than home-prepared foods now dominate American food consumption, everyday food and drink could represent a significant fluoride exposure. This matter has received some attention, and subsequent corrective action, with respect to infant formula; however, the issue goes beyond that. Meanwhile, our fluoride concentrations in drinking water, toothpastes, and other dental products remain much the same as they were when first developed years ago. One significant recognition of increased fluoride exposure came in 1994, however, when the American Dental Association significantly reduced its recommended schedule for fluoride dietary supplements.

With this widescale exposure to fluoride, it is hardly surprising that the prevalence of dental fluorosis is today much higher than it was 50 years ago. Most of the increase has been in the mildest forms of fluorosis, which are of questionable importance as a public health issue. But the trend toward a rising prevalence of fluorosis has been. fairly consistent over recent years, and the extent of the moderate-severe categories, while still only a small fraction of overall prevalence, has increased. Opinions differ on whether positive action should be taken to try to reduce the prevalence of fluorosis. One point of view says that the issue is not a public health problem, that most of those with very mild fluorosis do not even know they have it, that caries prevention is being maximized, and that drawing attention to the subject will only cause unnecessary political trouble. Another says that while fluorosis is hardly a public health issue now it could quickly become so; if this happened, many of the gains we have made with fluoride could be jeopardized. The consequence to this line of thought is therefore that steps should be taken to reduce overall fluoride ingestion before fluorosis becomes a major issue.

In August 1993, the National Research Council (NRC) released its report on the health effects of ingested fluoride (2). The NRC had been requested by the Environmental Protection Agency (EPA) to reexamine the EPA's upper limit of $4.0 \mathrm{ppm}$ fluoride for drinking waters. The report concluded that there was no evidence at present that would require this upper limit to be reduced, but that the matter should be constantly reviewed as new research findings became available. Fluoride, in the concentrations and amounts used and recommended in the United States, was seen as no hazard to human health. The report agreed with the EPA view that dental fluorosis, which was recognized as being associated with fluoride use, was more a cosmetic issue than a health problem. One area where the NRC did recommend further research, because of some conflicting reports in the literature, is in the area of fluoride intake and bone fractures, especially hip fractures in postmenopausal women.

Our first two speakers today will focus on two of these issues: that is, the ingestion of fluoride in the early years of life, and the relation between fluoride intake and bone strength in the later years. Dr. Steven Levy, of the University of Iowa, is the principal investigator for a research project, funded by the National Institutes of Health (NIH), to study the fluoride intake of infants and young children. Dr. Kathy Phipps, of the Oregon Health Sciences University, is the principal investigator on two NIH-funded projects investigating the relationship between fluoride intake and its effect on bone. The third speaker is Dr. Herschel Horowitz, who will give an overview on these and related issues from the perspective of his extensive experience in conducting fluoride clinical trials. We hope that the symposium will bring you some new information, and that it will stimulate your own thoughts on this important subject.

\section{References}

1.Mason JO. Too much of a good thing? Questions about fluorosis explored. J Am Dent Assoc 1991;122:93-6.

2.National Research Council, Committee on Toxicology. Health effects of ingested fluoride. Washington, DC: National Academy Press, 1993. 